\title{
CÂNCER DE PRÓSTATA: EPIDEMIOLOGIA E ASSOCIAÇÃO COM INFECÇÃO URINÁRIA
}

\author{
PROSTATE CANCER: EPIDEMIOLOGY AND ASSOCIATION WITH URINARY \\ TRACT INFECTION
}

\author{
Thiago Correa Bandeira, Jessica Deluca, Rubiane Beal, Claudinei Mesquita da Silva, Leyde \\ Daiane de Peder
}

Centro Universitário da Fundação Assis Gurgacz - FAG

\begin{abstract}
Prostate cancer is the second most common cancer among men in Brazil and mainly affects individuals over 65 years of age. The objective of the present study was to determine the epidemiological characteristics of patients with prostate cancer, as well as the prevalence of urinary infections in these patients. A descriptive and quantitative study carried out in medical records of adult patients attended from January 2015 to January 2016, in a reference hospital in cancer, located in Cascavel, Paraná. We analyzed 503 medical records of patients over 30 years of age. Of these, $46 \%$ were over 70 years of age, 76\% were white, 96\% had no higher education and $91 \%$ were treated by SUS. Urinary infection was observed in 29\% of the patients under treatment for prostate cancer, and Ciprofloxacin (28\%) was the main antimicrobial agent prescribed. It has been found that the number of prostate cancer cases has been increasing gradually in the studied population, becoming increasingly common in men mainly over 50 years, and that urinary infection may be related to prostate cancer due to changes cellular and subsequent risk of mutation.
\end{abstract}

Key words: Prostatic neoplasms; Urinary Tract Infections; Epidemiology

\section{Resumo}

O câncer (CA) de próstata é o segundo câncer mais comum entre os homens no Brasil e afeta principalmente indivíduos com idade superior a 65 anos de idade. 0 objetivo do presente trabalho foi determinar as características epidemiológicas de pacientes portadores de câncer de próstata, bem como a prevalência de infecções urinárias nestes pacientes. Pesquisa de caráter descritivo e quantitativo realizada em prontuários, de pacientes adultos atendidos no período de janeiro de 2015 a janeiro de 2016, em um hospital de referência em câncer, situado em Cascavel, Paraná (PR). Foram analisados 503 prontuários de pacientes com idade superior a 30 anos. Destes, $46 \%$ possuíam acima de 70 anos, 76\% eram de etnia branca, 96\% não possuíam ensino superior e $91 \%$ realizou tratamento pelo SUS. Infecção urinária foi observada em $29 \%$ dos pacientes em tratamento para câncer de próstata sendo que o principal antimicrobiano prescrito foi o Ciprofloxacino (28\%). Verificou-se que o número de casos de câncer de próstata vem aumentando gradativamente na população estudada, tornando-se cada vez mais comum em homens principalmente acima de 50 anos, e que a infecção urinária pode estar relacionada com o câncer de próstata devido às alterações celulares e posterior risco de mutação.

Palavras chave: Neoplasia de Próstata; Infecções Urinárias; Epidemiologia 
Introdução

O câncer é considerado um grande problema na saúde pública mundial, e está entre as principais causas de óbito em indivíduos adultos. Em 2008, ele foi responsável por aproximadamente 7,6 milhões de mortes em todo o mundo. Estima-se para o ano de 2030, 27 de milhões de casos e como consequência, 17 milhões de mortes e 75 milhões de pessoas vivendo com câncer sendo nos países que estão em desenvolvimento, a grande maioria dos casos $^{1}$.

O câncer de próstata é o segundo câncer mais comum entre os homens nos Estados Unidos $^{2}$. No Brasil, o Instituto Nacional do Câncer estimou que aproximadamente 69.000 novos casos dessa doença para 2014/2015, representando um aumento de aproximadamente $24 \%$ em comparação com $2010^{3}$. Além disso, a mortalidade relacionada à taxa de câncer de próstata mostrou um aumento preocupante em todas as regiões do Brasil, especialmente no Nordeste, onde a taxa aumentou de 3,8 mortes em 1980 para 14,3, por 100 mil homens em $2010^{4}$. Como a idade é o principal fator de risco associado ao câncer de próstata, o aumento na incidência e mortalidade desta patologia pode ser atribuído ao aumento da expectativa de vida ${ }^{5}$.

Os sintomas mais frequentes relacionados ao câncer de próstata são as disfunções miccionais como polaciúria, disúria, redução da força e calibre do jato urinário, noctúria, hesitação e a sensação de repleção miccional. Estima-se que a incidência de retenção urinária aguda seja de 13 a $25 \%$ em pacientes que desenvolveram câncer de próstata. Pode ser observado em estágios avançados, hematúria (devido à invasão da bexiga), obstrução uretral, com consequente hidronefrose e uremia, ou menos frequente, sangramento retal ${ }^{6}$. A prevenção é a principal aliada à redução da incidência e prevalência das doenças na população. Sabe-se que queda das taxas de morbidade e mortalidade pode estar diretamente associada com o reconhecimento inicial de doenças crônicas e mudança de hábitos $^{7}$. No entanto, o limitado conhecimento sobre a etiologia do câncer de próstata é um dos principais desafios associados ao desenvolvimento de medidas preventivas contra esta doença ${ }^{8}$.

A incidência de infecções do trato urinário (ITU) aumenta entre homens com idade superior a 50 anos $^{9}$ A alteração das vias urinárias, incluindo o uso de sonda vesical e a ocorrência de doença prostática são os principais fatores implicados no aumento da incidência no público masculino. Além disso, as taxas de ITU também são elevadas entre os idosos devido à presença de morbidades que aumentam a susceptibilidade às infecções, a partir disso, o objetivo do presente trabalho foi descrever as características epidemiológicas de pacientes portadores de câncer de próstata atendidos em um centro de referência em oncologia na região oeste do Paraná, bem como, relatar a epidemiologia da infecção urinária nestes pacientes. Estudos epidemiológicos constituem importantes ferramentas para subsidiar políticas e práticas de prevenção e promoção da saúde nos pacientes portadores de câncer de próstata.

\section{Metodologia}

Estudo de corte transversal, descritivo, com dados coletados em prontuários de pacientes atendidos em um hospital oncológico de referência no oeste do Paraná, localizado no município de Cascavel, no período compreendido entre 01 de janeiro de 2015 a 01 de janeiro de 2016, isto é um ano de estudo. Após aprovação no Comitê de Ética em Pesquisa do Centro Universitário da Fundação Assis Gurgacz, CAAE número 60699916.9 .0000 .5219 de 31 de outubro de 2016 e apresentação de uma Carta de Concordância do hospital de Câncer, foram incluídos todos os prontuários eletrônicos presentes nos arquivos digitais da unidade hospitalar.

Para caracterizar epidemiologicamente e clinicamente a população os dados ausentes do prontuário eletrônico, foram complementados utilizando os prontuários físicos de pacientes com faixa etária acima de 30 anos de idade, portadores de câncer de próstata atendidos no período definido para o estudo e residentes em Cascavel - PR e região. Foram analisados 503 prontuários que corresponderam aos critérios de inclusão (idade e residência).

As variáveis de interesse presentes nos prontuários e incluídas na análise foram: idade, etnia, profissão, escolaridade, convênio, data do diagnóstico, tipo de câncer, sintomatologia, comorbidades, infecções urinárias no último ano, medicamentos para infecção caso tenha utilizado, situação atual, exames laboratoriais.

Os dados coletados foram armazenados em Microsoft Excel e para análise e estudo sobre a associação entre variáveis coletadas dos prontuários adotou-se o teste de Qui Quadrado (teste $x^{2}$ ) em nível de significância de 5,0\%, com resultados considerados estatisticamente 
significativos para $p<0,05$. A análise estatística foi realizada através do software Bioestat, versão 4.

\section{Resultados}

De acordo com os resultados relativos às variáveis em estudo pode-se observar que o número de portadores de câncer de próstata vem aumentando ao longo os anos na população em estudo. Para a variável data do diagnóstico, verificou-se que muitos pacientes já eram portadores da doença no momento do atendimento realizado no período de estudo, verificando-se que o ano de diagnóstico relatado nos prontuários presentes no hospital oncológico variou de 2006 a 2015. No presente estudo foram observados picos de casos de câncer de próstata nos anos de 2010 (16\%) e 2015 (18\%), sendo que a partir de 2013 houve um aumento progressivo.

Figura 1. Ano do diagnóstico do câncer de próstata. Pacientes atendidos em um hospital de referência em câncer de Cascavel - PR, 2015.

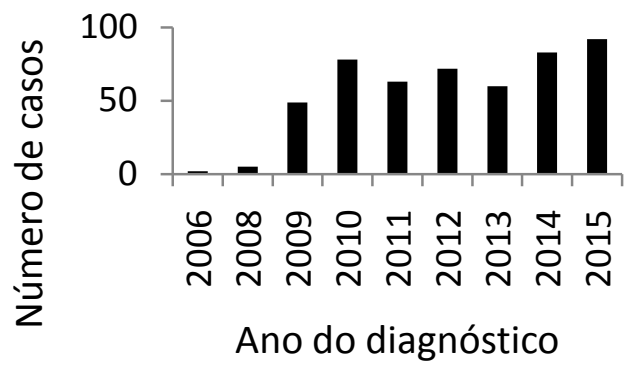

Tabela 1. Características epidemiológicas de pacientes portadores câncer de próstata atendidos em hospital de referência em câncer em Cascavel - PR, 2015

\begin{tabular}{|c|c|c|c|}
\hline Características epidemiológicas & $\mathbf{n}$ & $\%$ & $\begin{array}{l}\text { Valor de } \\
p\end{array}$ \\
\hline \multicolumn{4}{|l|}{ Idade (anos) } \\
\hline 30 a 40 & 1 & 0 & \multirow[t]{6}{*}{$<0,001$} \\
\hline 41 a 50 & 7 & 1 & \\
\hline 51 a 60 & 42 & 8 & \\
\hline 61 a 70 & 141 & 28 & \\
\hline 71 a 80 & 226 & 46 & \\
\hline$\geq 81$ & 86 & 17 & \\
\hline \multicolumn{4}{|l|}{ Etnia } \\
\hline Branca & 368 & 74 & \multirow[t]{4}{*}{$<0,001$} \\
\hline Parda & 97 & 20 & \\
\hline Negra & 30 & 5 & \\
\hline Outros & 8 & 1 & \\
\hline \multicolumn{4}{|l|}{ Educação } \\
\hline Não alfabetizado & 76 & 15 & \multirow[t]{5}{*}{$<0,001$} \\
\hline Ensino fundamental & 273 & 55 & \\
\hline Ensino médio & 86 & 17 & \\
\hline Ensino médio incompleto & 46 & 9 & \\
\hline Ensino superior & 22 & 4 & \\
\hline \multicolumn{4}{|l|}{ Fumante ou ex-fumante } \\
\hline Sim & 99 & 20 & \multirow[t]{2}{*}{$<0,001$} \\
\hline Não & 404 & 80 & \\
\hline \multicolumn{4}{|l|}{ Etilista ou ex-etilista } \\
\hline Sim & 45 & 9 & \multirow[t]{2}{*}{$<0,001$} \\
\hline Não & 458 & 91 & \\
\hline \multicolumn{4}{|l|}{ Profissão } \\
\hline Aposentado & 282 & 56 & \multirow[t]{3}{*}{$<0,001$} \\
\hline Agricultor & 89 & 18 & \\
\hline Demais & 132 & 26 & \\
\hline
\end{tabular}


...continuação

\section{Doença crônica}

Sim

Não

Sistema de saúde

SUS

Convênios/Particular

44

$91<0,001$

n, número de pacientes

A média de idade dos pacientes com CA de próstata foi de 72,6 anos. Verificou-se maior número de casos entre 71 a 80 (46\%) anos, seguido por pacientes entre 60 e 71 anos (28\%). A maioria dos homens (74\%) era etnia branca. Com relação à escolaridade, 273 (55\%) tinham o ensino primário, 86 (17\%) o ensino médio e 76 (15\%) não eram alfabetizados. Com relação ao estilo de vida, 99 pacientes declararam ser fumantes ou ex fumantes (20\%), e 45 (9\%) etilistas ou ex etilistas. Não houve diferença significativa entre os pacientes que não apresentavam doenças crônicas com relação aos que apresentavam $(p=0.920)$.

O número de pacientes que buscou tratamento pelo Sistema Único de Saúde foi significativamente maior que aqueles que optaram por convênios ou particulares. A maioria dos pacientes (282) apresentava como principal renda a aposentadoria.

Em relação à infecção do trato urinário, 29\% dos pacientes analisados por meio dos prontuários apresentaram no mínimo uma infecção ao ano, tendo em vista que a maioria faz consultas semestrais ou anuais, de acordo com o relatado pelos profissionais atuantes no hospital em estudo. Os principais antimicrobianos prescritos foram Ciprofloxacino (28\%), Norfloxacino (22\%) e Levofloxacino (13\%). As características relacionadas aos pacientes portadores de ITU estão descritas na Tabela 2.

Tabela 2. Características relativas à infecção urinária em pacientes portadores de câncer de próstata atendidos em hospital de referência em câncer em Cascavel - PR, 2015

\begin{tabular}{|c|c|c|c|}
\hline Características & $\mathbf{n}$ & $\%$ & Valor de $p$ \\
\hline \multicolumn{4}{|c|}{ Teve infecção urinária } \\
\hline Sim & 148 & 29 & $<0,001$ \\
\hline Não & 355 & 71 & \\
\hline \multicolumn{4}{|c|}{ Fez uso de medicamentos para infecção urinária } \\
\hline $\operatorname{Sim}$ & 215 & 43 & 0,193 \\
\hline Não & 288 & 57 & \\
\hline \multicolumn{4}{|c|}{ Medicamentos mais utilizados } \\
\hline Levofloxacino & 22 & 13 & $<0,001$ \\
\hline Ciprofloxacino & 55 & 28 & \\
\hline Norfloxacino & 43 & 22 & \\
\hline Nitrofurantoína & 17 & 9 & \\
\hline Cefalexina & 32 & 16 & \\
\hline Amoxicilina & 6 & 4 & \\
\hline Metronidazol & 5 & 3 & \\
\hline Outros & 9 & 5 & \\
\hline
\end{tabular}

n, número de pacientes 


\section{Discussão}

O câncer de próstata é o segundo câncer mais comum em homens, com incidência de 31,1 por 100.000 , e é a quinta causa mais comum de morte relacionada ao câncer ${ }^{10}$. Com exceção do aumento da idade, histórico familiar e raça ${ }^{11}$, os fatores etiológicos que desencadeiam o câncer de próstata ainda não foram totalmente identificados. Existem evidências que relacionam a inflamação à carcinogênese prostática ${ }^{12}$, pois a inflamação é resultante da exposição a agentes microbianos, que estimulam a produção de citocinas inflamatórias e espécies reativas de oxigênio, levando ao aumento da proliferação celular e possivelmente, à carcinogênese ${ }^{13}$. Essa relação já é comprovada nos cânceres do cólon, esôfago, estômago, bexiga e fígado ${ }^{14}$. Como a próstata é exposta a agentes infecciosos através da urina e da atividade sexual, a hipótese de que estes agentes podem iniciar processos inflamatórios e liderar a transformação maligna na próstata é razoável ${ }^{14}$.

Segundo Romero ${ }^{14}$ e Tortajada $^{9}$ o risco de câncer de próstata em negros apresenta um índice até $60 \%$ maior que brancos em países como EUA e Canadá. Nossos resultados podem ser justificados pelo fato da miscigenação existente no Brasil, onde outros casos estudados apresentaram valores de até $74 \%$ de pacientes brancos $^{9,15}$. O presente estudo mostrou que $74 \%$ dos homens eram de etnia branca, o que diverge do estudo de Pinheiro et $\mathrm{al}^{16}$ e Souza et $\mathrm{al}^{17}$, que verificaram maior incidência em etnias parda e negra respectivamente.

Com relação às variáveis idade e escolaridade, aproximadamente $70 \%$ se enquadram em não alfabetizados ou com ensino primário e $99 \%$ estão acima dos 50 anos de idade, o que vai de encontro ao estudo de Pinheiro et al $^{16,}$ o qual mostrou que $31,7 \%$ dos pacientes não continham instrução ou possuíam baixo nível de escolaridade e 55\% apresentaram idade superior a 55 anos. Souza et al $^{17}$ relataram que $65 \%$ dos pacientes possuíam o ensino fundamental e $100 \%$ apresenta idade superior a 67 anos. Dalbosco et $\mathrm{al}^{15}$ e Schor et al ${ }^{18}$ relatam que em homens adultos jovens as ITU são raras e quando ocorrem geralmente estão associadas com anormalidades urinárias estruturais. Por outro lado, após os 60 anos verificou-se um aumento de incidência que chega a atingir 3\% a $4 \%$ de homens e se relacionam com quadros de hiperplasia prostática, estreitamento uretral e outras anormalidades anatômicas.

Pinheiro et al $^{16}$ relataram em seu estudo, que $48 \%$ dos homens apresentaram doenças crônicas, $23 \%$ eram etilistas e $12 \%$ fumantes, ficando próximo aos resultados encontrados no nosso estudo, onde $51 \%$ eram doentes crônicos, $9 \%$ etilistas e $20 \%$ fumantes. O trabalho de Souza et $\mathrm{al}^{17}$ apresentou altos índices de pacientes fumantes, porém não existem estudos conclusivos que associem diretamente o cigarro como um causador do câncer de próstata.

Dos pacientes pesquisados, $29 \%$ relataram sintomas de infecção urinária em sua primeira consulta. Estudos como o de De Marzo ${ }^{19}$, relatam que a inflamação e a infecção geram espécies moleculares altamente reativas, como superóxido, peróxido de hidrogênio e óxido nítrico, que são liberados de células inflamatórias e podem danificar o DNA das células epiteliais, o que resulta na morte celular, ou seja, uma infecção pode evoluir para uma neoplasia.

Considerando que existem algumas evidências epidemiológicas que associam prostatite sintomática ao risco de câncer de próstata, as preocupações com os estudos de casos e controles da prostatite incluem um viés de detecção. Isto é, uma vez que a prostatite está associada ao aumento dos níveis de PSA, se um homem visitar o seu médico para sintomas de prostatite e verificar-se que ele tem um PSA elevado, provavelmente serão realizados exames para o câncer de próstata e, portanto, mais probabilidades de ser diagnosticado com o mesmo $^{19}$.

No presente estudo verificou-se que embora 148 pacientes tenham apresentado infecção urinária, 215 foram tratados com antibióticos. Isso normalmente se dá devido à profilaxia realizada com pacientes portadores de câncer devido ao risco de desenvolvimento de infecção, além disso, $28 \%$ dos pacientes que apresentaram a infecção, foram tratados com Ciprofloxacino, que é o medicamento de escolha para tratar prostatite bacteriana crônica e infecções relacionadas com o trato urinário, devido à sua boa atividade microbiana contra os agentes patogênicos que causam a maioria das infecções prostáticas ${ }^{20}$.

Ciprofloxacino pode inibir a inflamação e, portanto, levar à homeostase do compartimento celular dentro da próstata, além do que, sua boa penetração no tecido e fluído prostático foi relatada por muitos autores ${ }^{21,22}$. Existem razões que indicam que o Ciprofloxacino pode ser usado em terapias adjuvantes de certos cânceres ${ }^{23-25}$. A influência do Ciprofloxacino em células da próstata foi examinada por vários estudos. Trabalhos como os de El-Rayes et $\mathrm{al}^{26}$ e Aranha et $\mathrm{al}^{27}$, apresentaram $\mathrm{O}$ câncer de próstata resistente ao hormônio linha celular (PC-3), e 
mostraram que o Ciprofloxacino diminui a proliferação de células cancerígenas atuando de forma sinergética com o etoposido. Outros estudos demonstraram que o Ciprofloxacino inibe a proliferação de células cancerosas ${ }^{28}$. Pinto et al $^{28}$ mostraram que o Ciprofloxacino sensibiliza Células Refratárias de Câncer de Próstata Hormonal (HRPC) para doxorrubicina e tratamento sistêmico de docetaxel. Todos esses experimentos indicam que o Ciprofloxacino pode ser uma droga potencialmente profilática, podendo atuar potencialmente contra células de câncer de próstata e proteger contra a carcinogênese. Como resultado pode-se observar que a medicina pode estar usando esse medicamento como profilaxia do câncer de próstata.

Pode-se verificar que no caso do câncer da próstata, o antimicrobiano Ciprofloxacino pode estar sendo utilizado como uma peça chave muito importante na profilaxia para o câncer por apresentar além de características antimicrobianas, também atuar na inibição na inflamação da próstata. Vários estudos recentes demonstram o resultado cada vez mais efetivo como prevenção.

\section{Conclusão}

No presente trabalho, verificou-se que o número de casos de câncer de próstata aumentou com o decorrer do tempo, principalmente em indivíduos acima de 50 anos.

As principais características epidemiológicas relacionadas aos pacientes estudados foram: faixa etária compreendida entre 70 a 80 anos, etnia branca e baixa escolaridade. Esse estudo também evidenciou que a prevalência das infecções urinárias em pacientes com câncer de próstata é maior do que em indivíduos sem a patologia (43\%), sendo o Ciprofloxacino o principal antimicrobiano utilizado, além de que a infecção urinária pode estar diretamente relacionada com o desenvolvimento do câncer de próstata. Estudos como o presente ajudam na definição das características da população mais atingida pela doença em questão, o que é de suma importância na determinação de medidas de prevenção e controle para com essa população.

\section{Referências}

1. Instituto Nacional de Câncer José de Alencar da Silva (INCA). Disponível em: http://www.inca.gov.br/wcm/dncc/2015/dianacional-combate-cancer.asp. Acesso em: 15 de agosto 2018

2. Siegel R, Ma J, Zou Z and Jemal A. Cancer statistics, 2014. CA Cancer J Clin 2014;64 (1):9-29.

3. Facina T. Estimativa 2014 - Incidência de Câncer no Brasil. Rev Bras Cancerol 2014. 60 (1):63.

4. Conceição $M B$, Boing $A F$ and Peres KG. Time trends in prostate cancer mortality according to major geographic regions of Brazil: an analysis of three decades. Cad Saúde Pública 2014;30(3):559-566.

5. Brasil, Ministério da Saúde. Instituto Nacional de Câncer José Alencar Gomes da Silva (INCA). Estimativa 2016: incidência de câncer no Brasil. Rio de Janeiro. Disponível em: http://www.inca.gov.br/estimativa/2016/sintes e-de-resultados-comentarios.asp. Acesso em 14 de agosto de 2018.

6. Barnes RW. Carcinoma of the prostate: a comparative study of modes of treatment. J Urol 1940; 44(2):169-176.

7. Ministério da Saúde. A vigilância, o controle e a prevenção das doenças crônicas não-transmissíveis : DCNT no contexto do Sistema Único de Saúde brasileiro / Brasil. Ministério da Saúde - Brasília : Organização PanAmericana da Saúde, 2005. Disponível em: http://bvsms.saude.gov.br/bvs/publicacoes/DCN T.pdf. Acesso em: 14 de agosto de 2018.

8. Rhoden EL, Averbeck MA. Câncer de próstata localizado. Rev AMRIGS 2010; 54:92-9

9. Tortajada, JF Castell JG, Tornero OB, García, JAO. Factores de riesgo constitucionales em el câncer de próstata. Actas Urológicas Espanõlas 2011;35(5):282-288.

10. Gronberg H. Prostate cancer epidemiology. Lancet 2003; 361(9360):859-864.

11. Nelson WG, De Marzo AM, Isaacs WB. Prostate cancer. N Engl J Med 2003; 349(4):366381.

12. Coussens LM, Werb Z. Inflammation and cancer. Nature 2002; 420(6917):860-867.

13. Platz EA and De Marzo AM. Epidemiology of inflammation and prostate cancer. J Urol 2004; 171 (2 Pt 2): S36-S40.

14. Romero, FR. Fatores de risco para câncer de próstata em uma amostra da população de Curitiba, PR. Tese (Doutorado em Ciências da saúde) - Universidade Federal do Paraná. Curitiba, 2012.

15. Dalbosco, V.; Srougi, M. \& Dall'oglio, M. Infecções do Trato Urinário. Rev Bras Med 2003; 60(6):320-328.

16. Pinheiro, JTG, Araújo, MCAC, Barbosa, HA. Perfil dos Homens Participantes do Ensaio Comunitário Sobre Prevenção do Câncer de Próstata, Revista Bionorte 2015; 4(1):35-49. 
17. Souza, ARA, Alemida, SS, Oliveira, DC. Análise Estatística do Câncer de Próstata Por Meio da Regressão Logística. Rev Bras Biom 2013; 31(3):441-448.

18. Schor, N \& Heilberg, IP. Infecção do trato urinário: fisiopatologia e tratamento. In: Prado, F. C.; Ramos, J. \& Valle, R. J. Atualização terapêutica 2007: Manual Prático de Diagnóstico e Tratamento. 23. ed. São Paulo, Artes Medicas, 2007, 1167p.

19. De Marzo AM, Platz EA, Sutcliffe $S, X u$ J, Grönberg H, Drake CG, Nakai $Y$, Isaacs WB, Nelson WG. Inflammation in prostate carcinogenesis. Nat Rev Cancer 2007 7(4):256269.

20. Sohn DW, Han CH, Jung YS, Kim SI, Kim SW, Cho YH. Anti-inflammatory and antimicrobial effects of garlic and synergistic effect between garlic and ciprofloxacin in a chronic bacterial prostatitis rat model. Int J Antimicrob Agents 2009; 34(3):215-219.

21. Dan M, Golomb J, Gorea A, Braf Z, Berger SA. Concentration of ciprofloxacin in human prostatic tissue after oral administration. Antimicrob Agents Chemother 1986; 30(1):88-89. 22. Hoogkamp-Korstanje JA, van Oort HJ, Schipper JJ, van der Wal T. Intraprostatic concentration of ciprofloxacin and its activity against urinary pathogens. J Antimicrob Chemother 1985; 14(6):641-645.

23. Gurtowska N, Kloskowski T, Drewa T. Ciprofloxiacin criteria in antimicrobial prophylaxis and bladder cancer recurrence. Med Sci Monit 2010;16(10):RA218-223.

24. Kloskowski T, Gurtowska N, Drewa T. Does ciprofloxacin have an obverse or reverse? Pulm Pharmacol Ther 2010;23(5):373-375.

25. Kloskowski $\mathrm{T}^{1}$, Gurtowska N, Nowak M, Joachimiak R, Bajek A, Olkowska J, Drewa T. The influence of ciprofloxacin on viability of A549, HepG2, A375.S2, B16 and C6 cell lines in vitro. Acta Pol Pharm 2011;68(6):859-865.

26. El-Rayes BF, Grignon R, Aslam N, Aranha $\mathrm{O}$, Sarkar FH. Ciprofloxacin inhibits cell growth and synergises the effect of etoposide in hormone resistant prostate cancer cells. Int J Oncol 2002;21(1):207-211.

27. Aranha $\mathrm{O}$, Grignon $\mathrm{R}$, Fernandes $\mathrm{N}$, McDonnell TJ, Wood Jr DP, Sarkar FH. Suppression of human prostate cancer cell growth by ciprofloxacin is associated with cell cycle arrest and apoptosis. Int J Oncol 2003;22(4):787-794.

28. Pinto AC, Moreira JN, Simoes S. Ciprofoxacin sensitizes hormone-refractory prostate cancer cell lines to doxorubicin and docetaxel treatment on a achedule-dependent manner. Cancer Chemother Pharmacol 2009;64(3):445-454.

\section{Endereço para Correspondência}

Centro Universitário da Fundação Assis Gurgacz

Av. das Torres, 500 - Loteamento Fag, Cascavel PR

CEP.: 85806-095

e-mail: leydepeder@yahoo.com.br

Recebido em 22/12/2017

Aprovado em 04/09/2018

Publicado em 12/09/2018 